\title{
L'Homme des Glaces authentifié par l'archéologie moléculaire
}

En septembre 1991, un homme momifié était retrouvé dans un glacier des Alpes tyroliennes à 3200 m d'altitude avec des habits en cuir, une hache en cuivre, un poignard, un arc et des flèches. La datation au carbone $14\left({ }^{14} \mathrm{C}\right)$ de prélèvements de peau et d'os indiqua un âge de 5100 à 5300 ans, ce qui correspond au néolithique tardif [1]. L'homme surnommé «l'Homme des Glaces" avait entre 20 et 40 ans (d'après le degré de fermeture des sutures des os du crâne et l'usure des dents), mesurait entre 1,56 et $1,60 \mathrm{~m}$ et avait une capacité crânienne de 1500 à $1560 \mathrm{~cm}^{3}$ [2]. Le repliement du lobe de son oreille gauche indiquait que l'Homme des Glaces avait dû tomber d'épuisement puis mourir de froid sur place. La nature remarquablement intacte de toutes les parties de son corps résultait de la conjonction, d'une part, d'une momification (déshydratation) due à un vent chaud (le fohn) et, d'autre part, d'une position géographique privilégiée qui l'avait mis, dans le glacier, à l'abri du flot du glacier [2]. En effet, la pression d'un glacier est telle que, habituellement, les corps rejetés par les glaciers sont morcelés sur des dizaines de mètres carrés. La découverte de l'Homme des Glaces constitue donc une richesse archéologique sans précédent qui a soulevé un intérêt considérable de la communauté scientifique et du public. Toutefois, différentes rumeurs faisant allégation de fraudes ont circulé.

L'investigation par la génétique moléculaire de l'Homme des Glaces devait cependant pouvoir éclaircir certains points. Les comparaisons des séquences d'ADN du corps avec celle de populations contemporaines de$\mathrm{m} / \mathrm{s} n^{\circ} 12$, vol. 10 , décembre 94 vraient permettre de révéler son affiliation ethnique. L'étude moléculaire d'organismes tels que virus, bactéries, parasites associés au corps seraient d'un grand apport pour comprendre l'évolution de ces organismes. Dans une première étape vers ces objectifs, l'équipe de Svante Paabo de Munich a analysé l'état de conservation de l'ADN de l'Homme des Glaces et a déterminé la séquence du segment hypervariable non codant (anse D) de l'ADN mitochondrial à partir de plusieurs échantillons prélevés stérilement sur le corps [3]. Une partie d'un de ces échantillons avait servi pour la datation au ${ }^{14} \mathrm{C}$. Dans la majorité des cas, les travaux sur l'ADN archéologique se sont limités à l'ADN mitochondrial car son existence en un nombre important de copies (plusieurs centaines de mitochondries par cellule) augmente la probabilité de survie de quelques molécules face à la dégradation de l'ADN qui survient post-mortem. De plus, l'ADN mitochondrial est un outil de choix pour l'étude de la génétique des populations humaines $(\mathrm{m} / \mathrm{s}$ $n^{\circ} 11$, vol. 10, p. 1157).

Les auteurs ont retrouvé une relation inverse entre l'efficacité de l'amplification enzymatique in vitro (PCR: polymerase chain reaction) de l'anse D et la taille du produit amplifié, ce qui est classique en archéologie moléculaire (202 pb : bonne amplification; 287 pb: amplification moyenne ; $394 \mathrm{pb}$ : amplification à peine détectable; $540 \mathrm{pb}$ : pas d'amplification). A l'aide d'une technique de PCR quantitative, ils ont montré que, malgré leur méthode très performante d'extraction de l'ADN, on ne trouvait chez l'Homme des Glaces que 10 équivalents de génome nucléaire par gramme de tissu (soit $10^{6}$ fois moins que ce que l'on attend pour un ADN non dégradé). Lors du séquençage après clonage des produits d'amplification de 394 pb de l'anse D mitochondriale, de nombreuses substitutions ont été retrouvées, présentes sur plus d'un clone, qui n'étaient donc pas dues à des erreurs de la polymérase durant la PCR. Certaines de ces substitutions étaient différentes selon le type de prélèvement. L'homoplasmie de l'ADN mitochondrial étant la règle chez l'homme, cela indiquait que la momie avait été contaminée par de l'ADN de plusieurs individus, probablement ceux qui l'avaient manipulée après sa découverte. Il a été montré que l'ADN archéologique est sujet à la dégradation des bases pyrimidiques et que pouvait alors se produire une "PCR sauteuse " (jumping PCR). Lorsqu'un produit de PCR se termine prématurément à cause de l'altération d'une base pyrimidique, il saute sur une autre molécule matrice, sert d'amorce et permet la poursuite de la polymérisation, créant ainsi une recombinaison in vitro [4]. L'hypothèse des auteurs était donc que des recombinaisons in vitro se seraient produites durant la PCR entre des séquences de l'Homme des Glaces et celles d'hommes contemporains, créant ainsi des molécules hybrides. Cette hypothèse a été confirmée car le séquençage de petits fragments d'amplification (100 à $150 \mathrm{pb}$ ) ne comprenant pas de pyrimidines altérées n'a plus révélé d'ambiguités. Les auteurs ont alors montré que l'ADN de l'Hommes des Glaces différait par deux bases de la séquence de référence humaine actuelle, ce qui est en accord avec ce que l'on attendrait 
d'un échantillon datant de la période du néolithique tardif. Enfin, la comparaison de cette séquence avec celles de différents groupes ethniques indique que l'Homme des Glaces est le plus proche des populations du centre et du nord de l'Europe [3]. Ainsi donc, l'Homme des Glaces n'est pas un canular et l'extraordinaire richesse archéologique qu'il représente va pouvoir être calmement exploitée en dehors de l'hystérie médiatique qu'il avait provoquée lors de sa découverte.

E.D.

1. Bahn PC, Everett K. Iceman in the cold light of day. Nature $1993 ; 362$ : 11-2.

2. Seidler H, Bernhard W, Teschler-Nicola $M$, Platzer W, Nedden D, Henn R, Oberhauser A Sjovold T. Some anthropological aspects of the prehistoric tyrolean ice man. Science $1992 ; 258$ 255-7.

3. Handt O, Richards M, Trommsdorff M, Kilger C, Simanainen J, Georgiev O, Bauer K, Stone A, Hedges R, Schaffner W, Utermann G, Sykes B, Paabo S. Molecular genetic analyses of the tyrolean ice man. Science $1994 ; 264$ : 1775-8.

4. Paabo S, Irwin DM, Wilson AC. DNA Damage promotes jumping between templates during enzymatic amplification. I Biol Chem 1990 ; 265 : 4718-21.

\section{BRÈVES}

Réparation directe par la télomérase d'extrémités subtélomériques tronquées. L'extrémité du bras court du chromosome 16 est particulièrement propice à l'étude des mécanismes de troncation et de réparation par la télomérase. L'ensemble des gènes constituant le locus $\alpha$-globine est en effet très proche de cette extrémité ; leur structure est parfaitement connue, leur amputation se traduit par un phénotype $\alpha$-thalassémique clairement identifié. Un premier cas avait été décrit par le groupe de D.R. Higgs à Oxford en $1990(\mathrm{~m} / \mathrm{s}$ $n^{\circ} 10$, vol. 7 , p. 1091). La même équipe vient, par l'étude d'une série de troncations naturelles de ce même bras court du chromosome 16 (16p13.3), de caractériser le mécanisme de réparation et de stabilisation par lequel agit la télomérase [1]. Le point de cassure exact a été déterminé et analysé par séquençage. Dans les six cas, les chromosomes tronqués ont été stabilisés par l'addition directe à l'ADN non télomérique de la séquence répétitive hexamérique du télomère (TAAGGG)n. Aucun motif commun n'a été retrouvé entre les différents points de cassure. Dans cing cas sur six on constate que les trois ou quatre derniers nucléotides précédant ce point d'addition sont complémentaires et en phase avec la matrice supposée de la télomérase. Une telle complémentarité se retrouve statistiquement dans un ADN environ tous les 10 nucléotides, ce qui pourrait impliquer l'action minimale d'une exonucléase jusqu'au point de révélation du triplet nécessaire. Dans le sixième cas, cette courte complémentarité n'a pas été retrouvée, mais a été rétablie par l'addition de deux nucléotides «orphelins", qui n'appartiennent ni au chromosome d'origine ni à l'hexamère télomérique. Ces observations semblent donc confirmer qu'une réparation chromosomique par la télomérase peut se faire sur une extrémité tronquée, sans séquence particulière, et que cette réparation stabilise le télomère. Cette stabilité a été vérifiée par l'analyse détaillée de cinq méioses successives.

Les extrémités télomériques des différents chromosomes présentent souvent une densité élevée de gènes de structure. Des réarrangements non décelables en cytogénétique, du type de ceux qui ont été retrouvés dans ces cas d' $\alpha$-thalassémie, pourraient être responsables d'autres maladies génétiques humaines.

[1. Flint J, et al. Am J Hum Genet 1994 ; 55 : 505-12.] 